\title{
ANÁLISIS ACTUALIZADO SOBRE LA EFECTIVIDAD DE LAS OPCIONES TERAPÉUTICAS DEL REFLUJO VESICO-URETERAL PRIMARIO
}

\author{
Manuel Álvarez Ardura, Enrique de la Peña Zarzuelo, Virginia Hernández Cañas, Nasser Amaruch \\ García, José Manuel de la Morena Gallego y Carlos Llorente Abarca.
}

Servicio de Urología. Fundación Hospital Alcorcón. Madrid. España.

\begin{abstract}
Resumen.- OBJETIVO: El propósito principal de nuestro trabajo fue realizar una Revisión Sistemática (RS) sobre el manejo terapéutico del Reflujo Vésico Ureteral Primario (RVUP).

MÉTODOS: Para ello se ha realizado una RS de los artículos aparecidos en todas las bases de datos disponibles, aplicando unos criterios de inclusión y exclusión de calidad mínima imprescindible.
\end{abstract}

RESULTADOS: Se ha realizado lectura crítica de los artículos seleccionados y el estudio estadístico correspondiente de los datos agrupados, según el tipo de tratamiento y beneficios aportados, así como sus efectos indeseables.

CONCLUSIONES: El tratamiento médico y el quirúrgico presentan la misma efectividad en la resolución de los grados I,
II y III de RVUP, recomendándose el primero de ellos como tratamiento inicial tras el diagnóstico. El manejo endoscópico es igual de efectivo que la cirugía abierta, con menores efectos secundarios, sin haber encontrado diferencias entre las distintas sustancias analizadas para la inyección. Para los reflujos de alto grado $I V$ y $V$ no existen evidencias suficientes que aconsejen o desaconsejen cualquiera de los tratamientos. El tratamiento quirúrgico abierto, para cualquier grado de RVU, sólo presenta superioridad con respecto al tratamiento médico en el número de episodios de pielonefritis aguda durante el seguimiento. Esta afirmación no es posible aplicarla para el tratamiento endoscópico.

Palabras clave: Reflujo vesicoureteral primario. Quimioprofilaxis. Tratamiento endoscópico. Cirugía. Reflujo vesicoureteral.

Summary.- OBJECTIVES: The primary objective of this study is to perform a systematic review of the therapeutic management of primary VUR in pediatric urology.

METHODS: A systematic review of the articles published in all of the available databases has been performed, including scientific evidence-based medicine criteria. Inclusion criteria concerning basic quality of the articles were considered essential, as well as exclusion criteria to be able to reject the articles.

RESULTS: A critic reading of selected articles, and statistical study of grouped data was performed according to the type of treatment and benefits contributed by each treatment, and also to their undesirable effects.

CONCLUSIONS: The following Conclusions were drawn from the results obtained and from the analysis of the texts. Both medical and surgical treatment present similar effectiveness concerning resolution of gra-des I, II and III VUR, and the former one is the recom-mended initial treatment following 
diagnosis. Endosco-pic treatment is exactly as effective as open surgery for grades I, II and III with fewer undesirable secon-dary effects. There are no differences concerning the efficacy of the different injected substances. Not enough eviden-ces exist for degrees $I V$ and $V$ that may recommend or advise against any of the treatments. In any degree of VUR, open surgical treatment is superior as far as medi-cal treatment is concerned only regarding the number of acute pyelonephritis episodes during follow-up. This con-clusion cannot be applied on endoscopic treatment.

Keywords: Vesicoureteral reflux. Prophylaxis. Endoscopic treatment. Surgery

\section{INTRODUCCIÓN}

El Reflujo Vesico Ureteral Primario (RVUP) constituye un problema habitual en la práctica urológica pediátrica. Sin embargo, el manejo de esta patología es todavía un motivo de gran controversia. La profusión de estudios y la diversidad de opiniones sobre su manejo lo caracterizan y el RVUP se haya todavía rodeado de numerosos "mitos" que han tardado décadas en ser disipados. No es sorprendente que la mayoría de las recomendaciones terapéuticas se basen más en la opinión de expertos que en datos objetivos (1).

Lejos de implementar el conocimiento sobre el tema con una nueva serie más o menos larga de pacientes tratados, la justificación de nuestro trabajo se fundamenta en la búsqueda de la evidencia sobre este tema concreto. Realizamos una actualización de una revisión sistemática sobre el manejo terapéutico del RVUP en la población pediátrica, cubriendo los siguientes tipos de tratamientos: tratamiento farmacológico mediante quimioprofilaxis antibiótica; tratamiento quirúrgico mediante reimplante ureteral y tratamiento endoscópico mediante la inyección submeática.

Además, se desean alcanzar los siguientes objetivos secundarios: obtener un estimador promedio ideal (tratamiento idóneo) a partir de las estimaciones cuantitativas y cualitativas de los estudios individuales y comparativos para cada grado de RVU; y aclara incertidumbres sobre diversos aspectos de la patología no abordables desde la perspectiva de estudios aislados, pero que pueden examinarse en el contexto de un meta-análisis.

\section{ESTRATEGIA DE BÚSQUEDA}

Se hicieron búsquedas en MEDLINE (1966-agosto 2007), EMBASE (1988-agosto 2007), listas de referencias de artículos, búsqueda manual de literatura gris y guías clínicas de asociaciones, revisión en el registro Cochrane de RS y de Ensayos Controlados, búsqueda de ensayos adicionales en las listas de referencias de los artículos relevantes, utilizando el Sciencie Citation Index.

La sistemática utilizada para la búsqueda y selección de artículos, así como su evaluación posterior y análisis ha sido previamente descrita (2).

\section{Conflicto de intereses}

Nuestro trabajo no presenta en su génesis ni desarrollo ningún potencial conflicto de interés.

\section{RESULTADOS Y DISCUSIÓN: ANÁLISIS DE LOS DA- TOS EN RELACIÓN CON LA OPCIÓN TERAPÉUTICA}

\section{a) Disminución del grado de RVU}

\section{Tratamiento médico}

Los principales problemas encontrados en los artículos seleccionados han sido la dificultad para establecer similitudes dentro del concepto de éxito (resolución completa vs disminución del grado). Algunos trabajos se refieren al éxito por unidad renal y otros al éxito por paciente. Por último, resultó dificultoso extraer conclusiones de la tasa de resolución por año de seguimiento, pues en la mayoría de los casos el resultado es expresado de forma global según el tiempo medio de seguimiento para la serie. Otras series por su antigüedad no hacen referencia a la clasificación internacional del RVU.

Se ha definido la edad media de desaparición del RVU entre los 4,6 y los 6,8 años (3-5). Estas cifras no son igualmente aplicables en ambos sexos. Así está descrito como para grados bajos (I y II) en niñas de 10 años de edad se resolverán espontáneamente casi la totalidad de los casos antes de los 13 años, sin embargo, sólo el $50 \%$ de los niños cumplirá esta condición (6). Para los grados III y IV no se observará resolución espontánea de casi ningún caso a esta edad (7). Basado en los estudios de Arant, Skoog, Belman y Tamminen (3, 8-10), Elder (11) ha publicado las curvas de resolución para los grados I, II y IV así como para el grado III ajustado por edad de diagnóstico. Knudson et al en un trabajo recientemente publicado concluyen que la tasa de resolución espontánea del RVU es del 60\% (80\% para el grado I, 68\% para el grado II, $45 \%$ para el III y $17 \%$ para los grados IV/V), siendo el grado inicial, la edad, la capacidad vesical al diagnóstico y la historia de hidronefrosis prenatal factores pronósticos independientes para la resolución del RVU (12).

\section{Tratamiento quirúrgico abierto}

Los datos obtenidos se han agrupado por técnica quirúrgica y grado de reflujo tratado. El resultado final del procedimiento quirúrgico se ha podido obtener en el $95 \%$ de los pacientes analizados, especificado en 4556 tratamientos exitosos de los 4691 uréteres tratados $(97,1 \%)$. No en todos los trabajos analizados se obtienen resultados individualizados en función del grado de RVU que fue tratado, sin embargo se obtuvieron suficientes casos que permiten sacar conclusiones con un alto grado de evidencia.

Para el grado I, de las 88 unidades renales tratadas el resultado fue efectivo en todas ellas, para el grado $I I$ el resultado fue exitoso en 723 de las 730 unidades tratadas (99\%), en el grado III 757 de los 765 uréteres operados corrigieron posteriormente el RVU $(98,9 \%)$, en el grado IV 242 de los $249(97,1 \%)$ y en el grado V 72 de los 79 operados (91\%). 
Merece la pena considerar sin embargo que el resultado en grados severos varía mucho de unas series a otras del 25 (13) al $100 \%(14,15)$ y que se ve muy influido por el tipo de técnica, habiéndose encontrado un importante número de artículos en las que se asociaba a la técnica de reimplante algún tipo de modelaje ureteral, no precisándose en estos trabajos si se correspondían a casos de RVU de alto grado o megauréteres refluyentes, incluyéndose en estos casos las técnicas clásicas descritas por Starr (16), Kalicinsky (17) o Hendren (18).

En general, podemos afirmar que el resultado medio del tratamiento quirúrgico es del $97 \%$, siendo mayor para los grados I-III y algo menor para el grado IV, pero con valores por encima del $95 \%$. Los grados $V$ tienen un peor resultado pero por encima del $90 \%$. Cuando se asocia técnica de modelaje por presencia de megauréter y reflujo este baja hasta el $87,2 \%$

\section{Tratamiento endoscópico}

Se han recogido 29 trabajos referidos al tratamiento endoscópico del RVU (19 - 48) en los que se analizan las sustancias empleadas con un mayor número de individuos y los períodos de seguimiento más largo. Los seguimientos medios variaron entre 1 año y 17 , siendo la media superior a los 4 años. En todos se aplicó la técnica endoscópica descrita por O’Donnell y Puri (49).

\section{Politetrafluoruro de etileno (Teflon $®$ )}

La primera descripción en la literatura sobre su uso fue realizada en 1984 (49). Desde entonces su manejo se ha popularizado y las series han alcanzado un tiempo medio de seguimiento en algunos casos superior a 14 años. La mayor parte de los trabajos publicados aportan un número superior de casos a la serie global, aludiendo únicamente al grado de resolución del RVU y con escaso tiempo de seguimiento. Se ha establecido el porcentaje de infección urinaria postratamiento en un $2,3-3,6 \%$. Del total de las series, el 2,4\% precisó solucionar el RVU con una técnica de reimplante abierta. No se hace mención en ninguno de ellos al fracaso de la técnica por la asociación del síndrome de vejiga hiperactiva, entendiéndose en todos ellos como alteraciones clínicas pero sin comprobación urodinámica

\section{Polimetilsiloxano (Macroplastico®)}

Presenta tiempos de seguimiento más cortos por haberse incorporado a la práctica clínica más tarde. En este caso se ha descrito una asociación entre el fallo de la técnica y la coexistencia de alteraciones vesicales en el 45-50\%. Herz describe la aparición de un 5\% de reflujo contralateral tras la primera inyección así como un $4 \%$ de reintervenciones por vía abierta tras fracaso de la inyección.

3. Dextranomero del copolímero de ácido hialurónico (Deflux®)

Desde que la FDA aprobara su uso en el año 2001, su uso se ha ido en aumento hasta ser plenamente aceptada como tratamiento de primera línea. Lackren (50) con un seguimiento de 5 años sobre 221 pacientes (338 unidades renales) presenta unos resultados exitosos del
$77,5 \%, 77,8 \%, 62,5 \%$ y $66,6 \%$ para los grados II, III, IV y $\mathrm{V}$ respectivamente; resultados que obtiene en el $81 \%$ de la primera inyección, que se incrementa al $43 \%$ de la segunda y el $50 \%$ de la tercera.

Cappoza (51) en un estudio aleatorizado comparando tratamiento endoscópico con profilaxis antibiótica mostró un porcentaje de éxito del $69 \%$ frente al $38 \%$. El mismo autor en otro trabajo interpreta la mayoría de los fallos en el contexto de una disfunción vesical presente hasta en el 265 de los casos con una tasa global de éxito del $84 \%$ (52). Otros grupos, como el de la Mayo Clinic presentan resultados más discretos con un porcentaje de resolución por grados del $62,5 \%, 72,3 \%, 57,1 \%$ y $14,3 \%$ respectivamente (53).

\section{b) Cicatrices renales}

La correcta identificación de las pielonefritis agudas en los niños, la afectación y extensión del daño renal causado y la determinación de la posibilidad de reinfección siguen siendo fundamentales para determinar los riesgos a largo plazo, y las posibilidades de desarrollar hipertensión arterial e insuficiencia renal en el futuro.

Los aspectos diagnósticos de las pielonefritis agudas y de la afectación renal han generado numerosos trabajos, con grandes discrepancias entre ellos. Los primeros artículos aparecidos en los que se determina la existencia de cicatriz renal post-pielonefritis, anteriores a la década de los ochenta, están basados en estudios urográficos, diagnosticándose daños renales permanentes en un $5-20 \%$ (54). Actualmente y gracias a los estudios con DMSA (ácido dimercaptosuccinio) marcado con Tc99m se diagnostican un $41 \%$ de lesiones renales permanentes en riñones que han padecido pielonefritis (55). La ecografía renal presenta una baja sensibilidad $40 \%$, según Nguyen (56). En la gammagrafía renal con DMSA, el marcador se incorpora en un $60-70 \%$ a los túbulos renales y sólo un $2-5 \%$ es excretado por la orina, de tal manera que da una valoración fiel de la función global y relativa de cada unidad renal, habiéndose determinado su sensibilidad y especificidad para el diagnóstico de cicatriz renal tras pielonefritis en el 87 y $100 \%$ respectivamente (57-58).

Las alteraciones en el DMSA (hipocaptación del trazador) después de un episodio febril en niños con un rango de edad de 9 a 21,7 meses oscila entre el 52 y el $92 \%$, aunque posteriormente sólo quedan cicatrices permanentes en un 15-41\% (59-62). La gammagrafía renal además diagnostica la cicatriz renal tanto en niños con reflujo demostrado, como en aquellos en los que no se identifica este mediante cistografía miccional, aunque existe asociación significativa entre el grado de reflujo y la aparición de cicatrices (63-66). De acuerdo con esto podemos considerar que el RVU es un factor crítico para la aparición de cicatrices renales, pero no el único. Estos hechos han sido considerados por algunos autores lo suficientemente significativos como para plantear la gammagrafía renal con DMSA Tc $99 \mathrm{~m}$ como prueba inicial e incluso única en el diagnóstico de un episodio de infección urinaria febril en el niño (67-68). 
La influencia del sexo en la relación RVUP - ITUcicatrices en el riñón está todavía poco clara pero parece que, mientras que en los niños la aparición de cicatrices está directamente relacionada con el RVU, a menudo congénito en las niñas la aparición de lesiones está más relacionada con las ITUs con o sin RVU asociado, aunque éste sea un factor predisponerte (69).

La prevención de cicatrices renales es pues el principal objetivo en el tratamiento del RVU, debiéndose analizar con profundidad. Se han estudiado 5 trabajos prospectivos y randomizados que comparan los resultados del manejo terapéutico, médico o quirúrgico, con respecto a la aparición de nuevas cicatrices renales. En 4 de ellos estas se determinaron mediante urografía intravenosa y en uno de ellos con gammagrafía renal (70-74). Sin embargo, la mayoría de los trabajos hacen referencia a descripciones individuales de una de las dos ramas de tratamiento, por lo que hemos realizado las comparaciones de las dos con aquellos grupos de similar distribución de casos, grados y tiempo de seguimiento, obteniendo riesgos relativos combinados de las diferentes series.

Se han analizado 15 trabajos de tratamiento médico y 15 de tratamiento quirúrgico, que incluyen 5 de tratamiento endoscópico, cuyas dos substancias empleadas fueron el Teflon $®$ y el Macroplastico $®$. Los trabajos que hacen referencia a estudios prospectivos y randomizados entre tratamiento quirúrgico y médico no muestran diferencias estadísticamente significativas. Todos los trabajos comparan cirugía abierta frente a tratamiento médico con quimioprofilaxis. No se han encontrado estudios randomizados prospectivos que comparen el tratamiento endoscópico con el médico. Sobre un total de 806 niños (453 operados y 353 de tratamiento médico), seguidos entre 3,4 y 5 años, todos con grados de RVU de III a V, el tratamiento médico fue ligeramente superior al quirúrgico pero sin alcanzar la significación estadística $(11,3 \%$ frente a $12,6 \%$ de nuevas cicatrices).

Merece la pena destacar, sin embargo que la aparición de estas, se retrasa más en el tiempo en los pacientes tratados con quimioprofilaxis. El $80 \%$ de las lesiones en el grupo de tratamiento quirúrgico se producen en los 10 meses siguientes a la cirugía, mientras que en el grupo de tratamiento médico aparecen a lo largo de los 5 años posteriores al seguimiento (71). Son conocidas las profundas discrepancias encontradas entre las dos ramas, europea (72) y americana (73), del Estudio Internacional sobre Reflujo. Se analizó la frecuencia de aparición de nuevas cicatrices tras tratamiento, siendo el 15,7\% (médico) y $17,2 \%$ (quirúrgico) en la rama europea y el $21,5 \%$ (médico) y $31,4 \%$ (quirúrgico) en la Americana. Estos datos han sido posteriormente corroborados por Piepsz (75), sobre un grupo randomizado y prospectivo, seguido durante cinco años, de pacientes afectos de RVU de alto grado, $82 \%$ de los cuales presentaban alteraciones previas al inicio del tratamiento en el DMSA, sin encontrar diferencias significativas entre la opción médica y quirúrgica. Los 15 estudios no randomizados, referidos a los resultados de la opción médica (quimioprofilaxis), combinados entre si, muestran un riesgo relativo del 4,1\% [0 -24,7\%, I.C.:95\%] (8, 71, 76-87). Los 11 trabajos que analizan la aparición de nuevas cicatrices renales tras tratamiento quirúrgico, se refieren a procedimientos de reimplante ureteral transvesical $(71,74,78,88,89)$, y presentan unos porcentajes medios del 4,6\% [0-16,7\%, I.C.:95\%]. Se han analizado 6 trabajos que de forma individual describen la aparición de nuevas cicatrices tras tratamiento endoscópico $(21,22,28,30,33,90)$, siendo la media de $5,2 \%$ [0-12,5\%, I.C.:95\%].

Recientemente apareció un estudio multicéntrico, aleatorizado y controlado que comparaba la actitud expectante frente a la quimioprofilaxis antibiótica como prevención para la formación de nuevas cicatrices. Los autores encontraron que sólo un 5,9\% de los pacientes presentaba cicatrices tras un episodio de pielonefritis aguda al año del seguimiento. No encontraron diferencias significativas en los pacientes con RVUP con respecto a los que no lo presentaban. Tampoco hallaron diferencias entre el grupo en observación y el grupo en tratamiento médico (91).

\section{c) Crecimiento renal}

La impresión clínica, basada en algunos trabajos publicados en la década de los 70 (92-93), de que el RVU está asociado al retraso en el crecimiento renal, especialmente en los casos de RVU de alto grado $(94,95)$ ha motivado el análisis ulterior de esta variable, comparando las diferentes opciones terapéuticas. Sin embargo, la determinación del tamaño renal está afectada por algunos problemas, incluso en los individuos normales. Las pruebas utilizadas para su medición están sujetas a numerosas subjetividades y limitaciones técnicas. La ecografía, si bien se considera una buena técnica para la medición de órganos intrabdominales, está influida por la experiencia del examinador. No queda claro tampoco que el tamaño renal esté íntimamente relacionado con su función. No existe un patrón de la normalidad morfológica renal, existen riñones largos y delgados y otros cortos y gruesos, con la misma masa nefronal en definitiva.

El tamaño renal no depende únicamente del crecimiento del riñón afecto sino también del mecanismo compensador que establece el riñón contralateral sano, de las malformaciones congénitas asociadas, pero no producidas por el RVU, del número de episodios de infecciones urinarias sucedidas previas al diagnóstico de RVU, y a los cambios por nefropatía asociada al reflujo estéril prenatal (96), especialmente en niños con reflujo de alto grado. La mayoría de los artículos encontrados que hacen referencia a la influencia que sobre el tamaño renal tiene cada tipo de tratamiento analizan series retrospectivas sin un apropiado grupo control ni seguimiento prolongado.

Existen tres estudios que de forma prospectiva y randomizada, utilizan la urografía como método para determinar el tamaño renal, y con seguimiento superior a cinco años en dos de ellos $(71,73)$, y superior a diez en el tercero (97). Ninguno de ellos encuentra diferencias estadísticamente significativas a favor de ninguna de las dos opciones (médica o quirúrgica). No hemos encontrado ningún trabajo que de forma individual o comparada con tratamiento quirúrgico y/o médico analice los resultados del tratamiento 
endoscópico sobre el desarrollo renal. Las series de Chertin $(21,22)$ que ofrecen resultados a largo plazo (más de 10 años), en reflujos de alto grado, tratados mediante la inyección de politetrafluoroetileno (Teflon®), sólo contemplan la resolución del reflujo.

\section{d) Función renal}

La influencia del tratamiento seleccionado en la mejoría o estabilización de la función renal presenta el inconveniente de no ser valorable si la afectación es unilateral, aunque esta fuese severa. Sólo la función renal alterada por afectación bilateral podría verse mejorada tras aplicar el tratamiento, y sólo cuando esta fuese severa (98). Smellie (99) realiza un estudio prospectivo y randomizado entre pacientes afectos de RVU de alto grado bilateral con nefropatía severa, para comparar el tratamiento médico con el quirúrgico. Clasifica a los pacientes por edad e índice de filtración glomerular. Establece un seguimiento medio de cuatro años, y en un grupo de 48 pacientes del total de la serie durante más de diez años. Tras estimar la función glomerular mediante aclaramiento, urografía y DMSA, no encuentra diferencias estadísticamente significativas entre los dos grupos. Analizados individualmente los dos tipos de tratamiento, de una parte Scott describe mejoría en la función glomerular en los pacientes intervenidos mediante reimplante ureteral, especialmente en aquellos con afectación bilateral y cuya función glomerular pretratamiento era inferior a $50 \mathrm{ml} / \mathrm{min} / 1.73 \mathrm{~m}^{2}$. De otra parte Poulsen (100) usando el mismo tipo de determinación para la función renal, obtiene los mismos resultados con tratamiento antibiótico independientemente del grado de reflujo. Igualmente en estudios controlados no se ha apreciado beneficio en la función glomerular tras aplicar tratamiento quirúrgico. Tampoco hemos encontrado trabajos que analicen este aspecto tras aplicar tratamiento endoscópico. Únicamente Läckgren (50) refiere no haber encontrado empeoramiento de la función renal dentro de los pacientes de su serie, sin aportar datos específicos a este respecto.

\section{e) Infección urinaria}

La experiencia clínica nos ha demostrado que los pacientes diagnosticados de RVU y que han sido sometidos a tratamiento, bien de quimioprofilaxis, bien quirúrgico, siguen presentando infecciones urinarias en el seguimiento de su patología. La importancia de estas infecciones es su relación directa con la aparición de nuevas cicatrices renales que puedan comprometer la función renal futura o derivar en una hipertensión arterial.

Basados en estudios prospectivos que ya han sido analizados en el apartado correspondiente a cicatrices renales y opción terapéutica, hemos demostrado que no existen diferencias estadísticamente significativas a este respecto entre las dos opciones terapéuticas. Razonablemente si existe una relación directa entre las infecciones urinarias y la aparición de pielonefritis, y por lo tanto de daño real con cicatriz, no deberían existir tampoco diferencias significativas entre las dos opciones terapéuticas con respecto al número de infecciones urinarias.
Con la utilización del DMSA como prueba sensible y específica de afectación renal se han aclarado aspectos que vienen a modificar algunas pautas de actuación terapéutica. No todas las infecciones urinarias febriles comportan la aparición de una cicatriz renal, abriéndose así un nuevo abanico de patologías que afectando al tracto urinario superior y produciendo fiebre, no dañan al parénquima renal, tal es el caso de pielitis o ureteritis. Jakobson encuentra cambios en el DMSA en el $86 \%$ de los pacientes en el momento agudo, disminuyendo al $59 \%$ a los dos meses y al $37 \%$ a los dos años tras la infección. Además sólo el $25 \%$ de estos niños tenían asociado RVU, valores que se repiten para otros autores entorno al $39 \%$. Estos hechos estarían relacionados con factores de adherencia uroepitelial del huésped o factores de virulencia del microorganismo. De otro lado, ni siquiera las imágenes de hipocaptación obtenidas en las fases agudas de la pielonefritis, visualizadas incluso a los tres meses de la misma, se confirman en análisis posteriores a los seis meses. En este caso hechos como el tratamiento precoz, incluyendo fármacos antiflamatorios (101) disminuyen la progresión del daño renal. Se hace pues necesario determinar con evidencias científicas, la relación entre las infecciones urinarias, definida por bacteriuria con síntomas, y opción terapéutica para el RVU.

En el brazo europeo del Estudio Internacional de Reflujo, aplicado a grados III y IV, no se encontraron diferencias estadísticamente significativas con respecto al número de infecciones urinarias, $39 \%$ para la cirugía 38\% para el tratamiento médico. Se encontraron resultados muy similares en el brazo americano. Beetz (102) en un estudio sobre 158 pacientes seguidos durante 20 años tras el reimplante ureteral (que en el $82 \%$ de los casos se habían descrito infecciones febriles preoperatorias y $18 \%$ infecciones urinarias afebriles), encuentra que tras la observación continuaron teniendo infecciones predominantemente afebriles un $66 \%$, incluidas el $74 \%$ de las mujeres. El riesgo relativo de padecer una infección urinaria post-tratamiento quirúrgico frente al médico es de $0,97(0,79-1,19,1 . C .: 95 \%)$ sin poder encontrar diferencias significativas entre ambas opciones terapéuticas.

Con respecto al riesgo de padecer una pielonefritis Jodal encuentra un $9,3 \%$ dentro de su serie quirúrgica, comparada con el $21 \%$ de la serie médica. Weiss encuentra datos muy similares $(7,8 \%$ vs. $22,1 \%)$. Elo en un estudio de casos y controles sobre 40 pacientes encuentra un $72,5 \%$ dentro del grupo de controles (tratamiento médico) frente al $22,5 \%$ de los casos quirúrgicos. Hansson (103) en un estudio de quimioprofilaxis con sulfadiacina-trimetropin encuentra un tasa de pielonefritis en los 860 meses de seguimiento del $27 \%$. Beetz (102) en el estudio antes referido describe que los pacientes intervenidos por RVU siguieron manteniendo infecciones urinarias, afebriles en un $16 \%$ y febriles en un $38 \%$, el $82 \%$ de los cuales se repitieron más de una vez. Como nos recuerda Mansfield (104) estas infecciones seguidas a tan largo plazo pueden verse sesgadas por la incidencia de infecciones urinarias en la mujer con el inicio de las relaciones sexuales. El riesgo relativo se situaría en un $0,39(0,26-0,58 \%$, I.C.: $95 \%)$ a favor del tratamiento quirúrgico. De los trabajos revisados sobre tratamiento endoscópico del RVU destacan los resultados ofrecidos por Lipsky, con un 37\% de infecciones urinarias 
tras inyección de colágeno a los 4 años de seguimiento; el $9 \%$ de infecciones urinarias y el 3,5\% de pielonefritis en una serie de 225 inyecciones de Deflux® de Lackren y el 2,3\% de infecciones urinarias de la amplia serie de Puri sobre 717 uréteres de alto grado.

En definitiva en la comparación entre tratamiento médico y quirúrgico encontramos tras la revisión de la literatura tres hechos paradójicos:

1. No existe diferencias estadísticamente significativas con respecto al número de infecciones urinarias.

2. El tratamiento quirúrgico está relacionado con una menor incidencia de pielonefritis postoperatorias que el manejo médico con quimioprofilaxis.

3. Sin embargo, no existen diferencias estadísticamente significativas con respecto al desarrollo de nuevas cicatrices.

\section{f) Hipertensión arterial}

Las cicatrices renales diagnosticadas en los niños afectos de RVU se producen como consecuencia de daño post-infeccioso al parénquima, displasia renal asociada al reflujo o ambos. Estas son la mayor causa de hipertensión arterial en el niño y en el adulto joven y son las responsables del 25\% de las insuficiencias renales terminales en niños en Gran Bretaña (105). Se hace necesario determinar, a partir de los trabajos publicados, qué pacientes están en riesgo de padecer hipertensión arterial $y / o$ insuficiencia renal. Cuáles son los factores de riesgo evitables de aparición de hipertensión e insuficiencia renal. Qué pruebas son necesarias solicitar durante el seguimiento y durante cuánto tiempo. Qué tipo de tratamiento se debe aplicar para cada caso con el fin de evitar la aparición de nuevas cicatrices y por lo tanto disminuir la probabilidad de padecer hipertensión arterial. Es imprescindible encontrar trabajos que analicen los diferentes factores de forma individual y multivariable y que posean un tiempo de seguimiento lo suficientemente amplio como para establecer conclusiones sólidas.

Es fundamental establecer grupos de comparación homogéneos en cuanto al diagnóstico (grado de RVU), sexo, edad y tratamiento administrado. En este sentido no se ha encontrado ningún trabajo que haga referencia a las consecuencias que sobre la tensión arterial tiene el tratamiento endoscópico, por lo que todas las conclusiones se centran en la comparación del tratamiento médico con antibioterapia profiláctica frente al tratamiento quirúrgico abierto.

Para el análisis de los resultados de hipertensión arterial se ha tenido en cuenta la definición y clasificación de hipertensión arterial dada por la Academia Americana de Pediatría. Se han analizado 8 trabajos (106-110), el último de ellos prospectivo y randomizado, que analizan la incidencia de hipertensión arterial con un tiempo de seguimiento de media superior a 14 años, en un grupo homogéneo de pacientes de ambos sexos. La eliminación del reflujo, bien de forma espontánea o por tratamiento quirúrgico no evita la predisposición a padecer hiperten- sión arterial en el futuro. La hipertensión aparece casi exclusivamente en presencia de cicatrices renales. Esto obliga en cierta manera a mantener un seguimiento a largo plazo de los pacientes con nefropatía por reflujo, especialmente en aquellos con lesiones renales difusas y bilaterales. El riesgo relativo de padecer hipertensión arterial en los pacientes con lesiones renales por cicatriz postpielonefrítica es del 2,92 (intervalo de confianza al 95\%: 1,2-7,1) (11). La aparición de hipertensión arterial es independiente del grado de reflujo existente pre-tratamiento, de la persistencia del mismo y de la presencia de infecciones urinarias en el curso posterior al tratamiento quirúrgico. La aparición de hipertensión arterial severa se describe entre un 1,2 y $2,7 \%$ de las series $(109,111,112)$.

\section{g) Desarrollo pondoestatural}

Los trabajos clásicos de la década de los 70 mostraban una tendencia de los niños con RVU a tener una talla y peso inferior al percetil correspondiente para su edad (113) incluso reafirmados posteriormente por otros autores (114) especialmente en los niños que padecían infecciones urinarias.

Nuevamente se plantea la discusión sobre si alguno de los tratamientos escogidos para el RVU, médico o quirúrgico, modifican esta tendencia. Smellie (115) no encuentra diferencias estadísticamente significativas en la velocidad de crecimiento y percentil en un grupo de 114 niñas sometidas a quimioprofilaxis con cotrimoxazol y nitrofurantoina frente a un grupo control.

Merrell (1 16) y posteriormente Polito (117) en un estudio retrospectivo sobre 94 pre-púberes, 30 de los cuales presentaban cicatrices renales (17 bilaterales) en el DMSA, demostraba los beneficios del tratamiento quirúrgico.

Para comparar las dos opciones de tratamiento entre si, se ha analizado cinco trabajos prospectivos. El trabajo prospectivo y randomizado entre tratamiento quirúrgico y médico realizado por Smellie y cols, seguidos durante 10 años no encuentra diferencias estadísticamente significativas en este aspecto. Igualmente se han obtenido los mimos resultados en la rama europea del estudio internacional de reflujo tal y como ha descrito Olbing. Otros estudios prospectivos anteriores a más corto plazo de seguimiento habían demostrado este mismo hecho $(118,119)$.

\section{h) Complicaciones asociadas al tratamiento quirúrgico}

\section{Obstrucción ureteral}

Esta complicación es la más frecuentemente descrita entre los textos seleccionados para esta revisión. Se han encontrado 51 artículos en los que se describe dicha complicación, 35 correspondientes a reimplantes con cirugía abierta, transvesical y extravesical $(120,121)$ y 18 correspondientes a tratamientos endoscópicos, utilizando gran variedad de sustancias para la inyección, (122-128). El índice de obstrucciones ureterales post-reimplante se encuentra entre el 0,9 y el $2 \%$, siendo las series anteriores a 1986 las 
que presentan una mayor tasa de obstrucción, tal y como se ha descrito en la revisión realizada por el grupo de expertos de la Asociación Americana de Urología. No se han encontrado diferencias significativas con respecto a la técnica quirúrgica practicada, tanto transvesical como extravesicalmente. La tasa de reoperaciones requerida para solucionar esta obstrucción ha variado igualmente del 0,3 al 9,1\%, lo que supone una incidencia media de reintervenciones por obstrucción tras reimplante ureteral del $2 \%$.

De los 489 uréteres tratados mediante inyección con polidimetilsiloxano (129), sólo en un caso de la serie de Al-Hunayan, se produjo obstrucción post-inyección siendo necesario el reimplante. En el estudio más amplio que conocemos de Puri, sobre un total de 53 urólogos de 41 centros diferentes así como en otro estudio del mismo autor (130) 3 años antes, en el ámbito europeo, sobre un total de 8.332 niños tratados con diferentes grados de RVU (I: $3,3 \%$, II: $31,2 \%$, III: $42,5 \%$, IV: $18,1 \%$ y $\mathrm{V}: 4,7 \%$ ), con una tasa de éxito global del $89 \%$ se describieron 41 obstrucciones ureterales post-inyección $(0,33 \%)$ que requirieron reimplante ureteral. Stenberg (131) en su serie utiliza las microesferas de dextrómero de ácido hialurónico sódico (Deflux system $\circledR$ ) y no encuentra casos de obstrucción ureteral postinyección.

\section{Hemorragia}

No se han encontrado diferencias significativas entre las diferentes técnicas quirúrgicas utilizadas en cirugía abierta siendo los casos de sangrado (hemorragia o hematoma) del $0,26 \%, 1,2 \%$. No se ha encontrado el sangrado como complicación en ninguno de los trabajos seleccionados y revisados sobre tratamiento endoscópico.

\section{Infección de herida quirúrgica}

La tasa de infección de herida quirúrgica, tras reimplante ureteral, ha sido descrita entre el 0,8 y el $1,2 \%$ $(132,133)$. No se han encontrado diferencias significativas entre los procedimientos transvesicales y extravesicales.

\section{Alteraciones funcionales postoperatorias}

Las alteraciones miccionales tras el reimplante ureteral han sido descritas no sólo desde el punto de vista clínico, sino incluso con modelos anatómicos que demuestran la posible afectación del plexo hipogástrico situado cercano a la unión urétero-vesical y que puede ser lesionado durante la desinserción ureteral (134). Especialmente este hecho se ha descrito en los reimplantes ureterales extravesicales (135) en la que se produce una sección longitudinal del detrusor vesical, y en concreto en los pacientes que presentaban antecedentes de trastornos funcionales, o en los que se realiza cirugía bilateral en un solo tiempo quirúrgico. Para la cirugía extravesical tipo Lich- Gregoir se ha descrito una incidencia de alteraciones funcionales significativas, que requirieron tratamiento en un 6,5\% (136). En esta serie un 2,5\% fueron tratados con anticolinérgicos y un $4 \%$ con cateterismos vesicales o sondaje permanente entre 2 y 21 días. Las alteraciones funcionales en los pacientes tratados mediante inyección endoscópica han sido analizadas en la mayoría de los trabajos como causa de fallo de la técnica y no como consecuencia de la misma. Cappozza describe un $4,3 \%$ de pacientes que presentaban disfunciones miccionales no conocidas previamente tras la inyección endoscópica que no requirieron tratamiento postoperatorio. Llama la atención como ninguna de las series revisadas basa en estudios urodinámicos las hiperactividad vesical descritas en sus series, que en la totalidad de los casos son definidas como alteraciones clínicas en el patrón miccional, con cuadros de urgencia miccional y urgencia - incontinencia. Serían necesarios más estudios en este sentido que pudiesen aportar evidencias significativas en cuanto a la aparición de alteraciones funcionales, pues posiblemente estén infravaloradas. No hemos encontrado así mismo ningún trabajo que compare de manera randomizada esta complicación entre el tratamiento quirúrgico abierto y el endoscópico.

\section{Aparición de RVU contralateral}

Este hecho ha sido sobradamente descrito en casos de aparición de RVU contralateral "de novo", así como de reaparición de un RVU contralateral solucionado espontáneamente (137). Se ha aludido a la existencia de un mecanismo de contra- presión, denominado "pop-off" que explicaría la aparición de un RVU de novo cuando la presión intravesical aumentase al suprimirse el RVU conocido. Este hecho no ha podido demostrarse tras procedimientos endoscópicos en RVU de alto grado (138). No se ha encontrado relación entre la aparición de reflujo contralateral y la edad, o grado de reflujo (139). Se ha descrito igualmente la aparición de RVU ipsilateral en el caso de duplicidades completas, aunque la mayoría de ellas no han necesitado de tratamiento quirúrgico para su resolución, siendo ésta espontánea (140). Elder y cols. han determinado el porcentaje estimado de RVU contralateral para diferentes grados de RVU intervenido $11,52 \%$ para el grado I y II, 2,8\% para el grado III, 3,66\% para el grado IV y 2,53 para el grado V) y diferentes técnicas quirúrgicas empleadas, no encontrando tampoco en éstas, diferencias estadísticamente significativas. No se encontraron diferencias significativas con respecto al tratamiento endoscópico por si sólo ni analizando el grado de reflujo preoperatorio o el material usado para la inyección.

\section{Dolor postoperatorio}

Se trata de uno de los efectos indeseables más difícilmente cuantificables a pesar de que se han utilizado varias escalas de dolor, aplicadas al control analgésico postoperatorio en niños. Se han descrito diferentes pautas de tratamiento como el bloqueo caudal con un periodo efectivo de analgesia de 12 horas postinyección sin presentar complicaciones relacionadas con el procedimiento, el uso de catéteres epidurales con analgesia continua, que se mantienen durante el periodo de cateterización vesical, disminuyendo la cantidad de analgésicos administrados durante el postoperatorio, pero con una incidencia mayor de episodios febriles postquirúrgicos, una estancia media similar a los analgésicos parenterales habituales y un mayor número de complicaciones asociadas al propio catéter epidural (141). También se ha descrito el uso de ketokerolato intravenoso como tratamiento analgésico para controlar los espasmos vesicales derivados del uso del catéter vesical, estableciéndose su seguridad y efectividad por encima del tratamiento placebo (142). Por último se ha utilizado la analgesia intravesical con mórficos, que igualmente reduce los espasmos vesicales tan mal tolerados por el niño. Sin embargo El- Ghoneimi (143) demuestra en un estudio 
controlado y randomizado que no existen diferencias significativas entre este y el placebo. No hemos encontrado datos objetivos que avalen la teoría de que el reimplante extavesical o la cirugía laparoscópica disminuyen el dolor postoperatorio comparado con el resto de procedimientos quirúrgicos.

\section{Días de hospitalización postoperatoria}

Se han analizado 10 estudios (144-147) en los que de forma principal ha sido analizado este parámetro, sobre un total de 637 pacientes, todos ellos realizados con cirugía abierta de reimplante ureteral. La estancia media osciló entre 2,4 días y 13,9 días aunque parece variar en función de la técnica usada (menor para las técnicas exravesicales que para las de reimplante intravesical) y de si se dejan catéteres ureterales o no $(5,4-5,6$ días frente a los 2,7-4,2 días respectivamente). Se han podido analizar varios trabajos en los que se realiza de forma ambulatoria o con ingresos inferiores a 24 horas de hospitalización el tratamiento endoscópico del reflujo, con diversas sustancias $(148,149)$.

\section{i) Complicaciones asociadas al tratamiento médico}

Las complicaciones derivadas directamente del manejo médico se refieren especialmente a aquellas relacionadas con los estudios radiológicos, tanto convencionales como isotópicos, es decir: radiaciones ionizantes, reacciones alérgicas al contraste yodado y cateterismos uretrales.

\section{Radiaciones ionizantes}

La Comisión Europea y el Ministerio de Sanidad y Consumo nos recuerdan a través de la guía de indicaciones para la correcta solicitud de pruebas de diagnóstico por la imagen (150) que aunque las ventajas de la realización de este tipo de pruebas compensan con creces los leves riesgos de la radiación, ni siquiera las pequeñas dosis están totalmente exentas de riesgos.

La cistografía isotópica directa es realizada con Tc $99 m$ en cloruro sódico al $0,9 \%$ en un medio estéril y apirógeno. No se conocen contraindicaciones asociadas al propio isótopo y no hemos encontrado estudios en animales que demuestren su potencial carcinogénico o alteraciones en la fertilidad.

Las dosis recomendadas para el estudio de RVU son de 18,5 a $37 \mathrm{MBq}(0,5-1 \mathrm{mCi})$, siendo las dosis de radiación absorbidas en niños. No se han descrito reacciones anafilácticas con el uso de la cistografía isotópica.

Para realizar las cistografías isotópicas directas y convencionales es necesario someter al paciente a cateterismo transuretral del cual se derivan complicaciones determinadas en un $35 \%$ de los casos, en forma de sintomatología miccional irritativa e infecciones urinarias (151). Aunque los primeros síntomas no parecen ser mejorados con la profilaxis antibiótica $34 \%$ con antibióticos frente al $37 \%$ sin antibióticos), ésta se hace imprescindible para evitar la infección urinaria. No se han encontrado docu- mentos que recojan las alteraciones psicológicas en los pacientes sometidos a cateterismo uretral para realizar una cistografía pero la opinión expresada por los urólogos infantiles y que han reflejado sin datos algunos de ellos (152) es que para muchos niños el cateterismo supone un suceso traumático, que condiciona en muchas ocasiones la repetición de la prueba durante el seguimiento de un RVU. Las reacciones alérgicas derivadas del uso de contraste yodado son infrecuentes en la población infantil, un $6 \%$ consideradas menores (náuseas, vómitos, urticaria, prurito o flash y cefalea) (153), y un $0,5 \%$ graves, pero sin haberse descrito muertes por reacciones alérgicas al contraste yodado (154).

\section{Reacciones adversas medicamentosas}

La quimioprofilaxis antibiótica que se utiliza en el tratamiento médico continuado se administra a razón de 1/4 o 1/3 de la dosis terapéutica completa. Los fármacos habitualmente utilizados y recogidos en la literatura científica son: el trimetopim, el cotrimoxazol (sulfametoxazoltrimetopim), la nitrofurantoína, y las cefalosporinas de primera y segunda generación por vía oral (cefonicid, cefalexina y cefazolina).

Las bajas dosis administradas y la comprobada seguridad del fármaco en niños producen muy bajos índices de efectos secundarios y son escasamente recogidos en la literatura. De una parte estos efectos secundarios son habitualmente menores (mal sabor de boca). De otra parte muchos de ellos son difícilmente achacables únicamente al fármaco como dolores abdominales inespecífi$\cos$ y difusos. No es frecuente encontrar referidos en los trabajos de tratamiento médico abandonos de la medicación por estos efectos, y la mayoría de los graves efectos secundarios se describen en adultos y en tratamientos a dosis plenas.

En cualquier caso la prolongación en el tiempo del tratamiento antibiótico es una inquietud habitualmente referida por los padres que puede condicionar el tratamiento a seguir. Los fármacos anticolinérgicos utilizados también en el tratamiento médico del reflujo asociado a inestabilidad vesical son el cloruro de trospio, la oxibutinina y la tolterodina. La fiabilidad de los tres ha sido probadamente comprobada tanto en adultos como en niños. Merece la pena destacar que, en nuestra experiencia, y como se recoge en la literatura la tolerabilidad del fármaco es mucho mayor en los niños que en los adultos. Al igual que el tratamiento antibiótico, la mayoría de los artículos revisados hacen referencia a efectos secundarios aparecidos en los adultos, en especial con la tolterodina, fármaco que en un principio no fue evaluado en la edad infantil, y del que ya se tienen evidencias sobre su seguridad y efectividad en los niños $(155,156)$.

\section{CONCLUSIONES DE LA REVISIÓN}

Tanto el tratamiento médico como el quirúrgico presentan la misma efectividad en la resolución de los grados I, II y III de RVU, recomendándose el primero de ellos como tratamiento inicial tras el diagnóstico. Grado de recomendación A. 
El tratamiento endoscópico es igual de efectivo que la cirugía abierta para los grados I, II y III. Grado de recomendación $\mathrm{A}$.

La incidencia de efectos indeseables secundarios al tratamiento endoscópico es menor que la cirugía abierta. Grado de recomendación A.

Para los grados I, II y III, el tratamiento quirúrgico recomendado es la inyección endoscópica, no existiendo diferencias en cuanto a la efectividad de las distintas sustancias. Grado de recomendación B.

Para los grados IV y $\mathrm{V}$ no existen evidencias suficientes que aconsejen o desaconsejen cualquiera de los tratamientos. Grado de recomendación C.

El tratamiento quirúrgico abierto, para cualquier grado de RVU sólo presenta superioridad con respecto al tratamiento médico en el número de episodios de pielonefritis aguda durante el seguimiento. No existen diferencias entre ambos para: las infecciones urinarias, la hipertensión arterial, la insuficiencia renal, el crecimiento renal, el crecimiento del niño ni la morbilidad gestacional. Grado de recomendación $\mathrm{A}$.

Esta misma conclusión no es posible aplicarla con los datos publicados para el tratamiento endoscópico. Grado de recomendación $\mathrm{C}$.

\section{BIBLIOGRAFÍA Y LECTURAS RECOMENDADAS ("lectura de interés $y^{* *}$ lectura fundamental)}

1. GARIN, E.H.; CAMPOS, A.; HOMSY, Y.: "Primary vesicoureteral reflux: Review of current concepts". Pediatr. Nephrol., 12: 249, 1998.

**2. DE LA PEÑA, E.: "Tratamiento del reflujo vesicoureteral primario en la infancia: Comparación de dos revisiones sistemáticas". Actas Urol. Esp., 29: 138, 2005.

3. SKOOG, S.J.; BELMAN, A.B.; MADJ, M.: "A non surgical approach to the management of primary vesicoureteral reflux". J. Urol., 138: 941, 1987.

4. SKOOG, S.J.; BELMAN, A.B.: "Primary vesicoureteral reflux in the black child". Pediatrics, 87: 538, 1991.

5. DUCKETT, J.W.: "Vesicoureteral reflux: A "conservative" analysis". Am. J. Kidney Dis., 3: 139, 1983.

6. GOLDRAICH, N.P.; GOLDRAICH, I.H.: "Follow up of conservatively treated children with high and low grade vesicoureteral reflux: A prospective study". J. Urol., 148: 1688, 1992.

7. LENAGHAN, D.; WHITAKER, J.G.; JENSEN, F. y cols.: "The natural history of reflux and long term effects of reflux on the kidney". J. Urol., 115: 728, 1976.

8. ARANT, B.S.: "Medical management of mild and moderate vesicoureteral reflux: Follow up studies of infants and young children. A preliminary report of the Southwest Pediatric Nephrology Study Group". J. Urol.,148: 1683, 1992.

9. BELMAN, A.B.; SKOOG, S.J.: "Non surgical approach to the management of vesicoureteral reflux in children". Pediatr. Infect Dis. J., 8: 556, 1989.
10. TAMMINEN-MOBIUS, T.; BRUNIER, E.; EBEL, K.D. y cols.: "Cessation of vesicoureteral reflux for 5 years in infants and children allocated to medical treatment. The International Reflux Study in children". J. Urol., 148: 1662, 1992.

**11. ELDER, J.S.; PETERS, C.A.; ARANT, B.S. y cols.: "Pediatric Vesicoureteral Reflux Guidelines Panel summary report on the management of primary vesicoureteral reflux in children". J. Urol., 157: 1845, 1997.

12. KNUDSON, M.J.; AUSTIN, J.C.; McMILLAN, Z.M. y cols.: "Predictive factors of early spontaneous resolution in children with primary vesicoureteral reflux". J. Urol., 178: 1684, 2007.

13. SOLOK, V.; EROZENCI, A.; KURAL, A. y cols.: "Correction of vesicoureteral reflux by the Gil-Vernet procedure". Eur. Urol., 14: 214, 1988.

14. GLASSBERG, K.I.; LAUNGANI, G.; WASNICK, R.J. y cols.: "Transverse ureteral advancement technique of ureteroneocistostomy (Cohen reimplant) and a modification for difficult cases (experience with 121 ureters)". J. Urol., 134: 304, 1985.

15. MATSUMOTO, F.; TOHDA, A.; SHIMADA, K.: "Effect of ureteral reimplantation on prevention of urinary tract infection and renal growth in infants with primary vesicoureteral reflux". Int. J. Urol., 11: 1065, 2004.

16. STARR, A.: "Ureteral placation: a new concept in ureteraltapering for megaureter". Invest. Urol., 17: 153, 1979.

17. KALICINSKI, Z.; KANSY, J.; KOTARBINSKA, B.: "Surgery of megaureters: Modification of Hendren's operation”. J. Pediatr. Surg., 12: 183, 1977.

18. HENDREN, W.H.: "Operative reapir of megaureter in children". J. Urol., 101: 491, 1969.

*19. SERRANO DURBÁ, A.; ESTORNELL MORAGUES, F.; DOMÍNGUEZ HINAREJOS, C. y cols.: "Eficacia del tratamiento endoscópico del RVU primario en la infancia con polidimetilxilosano". Urol. Int. Invest., 7: 490, 2002.

20. FARKAS, A.; MURIEL, E.Z.; LUPA, S.: "Endoscopic correction of vesicoureteral reflux: Our experience with 115 ureters". J. Urol., 144: 534, 1990.

**21. CHERTIN, B.; COLHOUN, M.; VELAYUDHAM, M. y cols .: "Endoscopic treatment of vesicoureteral reflux: 11 to 17 years of follow up". J. Urol., 167: 1443, 2002.

22. CHERTIN, B.; DE CALUWE, D.; PURI, P.: "Endoscopic treatment of primary grades IV and $\mathrm{V}$ vesicoureteral reflux in children with subureteral injection of polytetrafluoroethylene". J. Urol., 169: 1847, 2003.

*23. STENBERG, A.; LARSSON, E.; LACKGREN, G.: "Endoscopic treatment with dextranomer-hyaluronic acid for vesicoureteral reflux: Histological findings". J. Urol., 169: 1109, 2003.

24. BROWN, S.: "Open versus endoscopic surgery in the treatment of vesicoureteral reflux". J. Urol., 142: 499, 1989.

25. ABOUTALEB, H.; BOLDUC, S.; UPADHYAY, J. y cols.: "Subureteral polydimethylsiloxane injection versus extravesical reimplantation for primary low grade vesicoureteral reflux in children: A comparative study". J. Urol., 169: 313, 2003.

26. HERZ, D.; HAFEZ, A.; BAGLI, D. y cols.: "Efficacy of endoscopic subureteral polydimethylsiloxane injec- 
tion for treatment of vesicoureteral reflux in children: A North American clinical report". J. Urol., 166: 1880. 2001.

27. AL-HUNAYAN, A.A.; KEHINDE, E.O.; ELSALAM, M.A. y cols.: "Outcome of endoscopic treatment for vesicoureteral reflux in children using polydimethylsiloxane". J. Urol., 168: 2181, 2002.

28. LIPSKY, H.; WURNSCHIMMEL, E.: "Endoscopic treatment of vesicoureteric reflux with collagen. Five years' experience". Br. J. Urol., 72: 965, 1993.

29. CAPOZZA, N.; CAIONE, P.: "Dextranomer/hyaluronic acid copolymer implantation for vesico-ureteral reflux: A randomized comparison with antibiotic prophylaxis". J. Pediatr., 140: 230, 2002.

30. PURI, P.; GRANATA, C.: "Multicenter survey of endoscopic treatment of vesicoureteral reflux using polytetrafluoroethylene”. J. Urol., 160: 1007, 1998.

31. HAFERKAMP, A.; MOHRING, K.; STAEHLER, G. y cols.: "Long-term efficacy of subureteral collagen injection for endoscopic treatment of vesicoureteral reflux in neurogenic bladder cases". J. Urol., 163: 274, 2000

32. CALDAMONE, A.A.; DIAMOND, D.A.: "Long-term results of the endoscopic correction of vesicoureteral reflux in children using autologous chondrocytes". J. Urol., 165: 2224, 2001.

33. OSWALD, J.; RICCABONA, M.; LUSUARDI, L. y cols.: "Prospective comparison and 1-year follow-up of a single endoscopic subureteral polydimethylsiloxane versus dextranomer/hyaluronic acid copolymer injection for treatment of vesicoureteral reflux in children". Urology, 60: 894, 2002.

34. VEREECKEN, R.L.; PROESMANS, W.: "Results of endoscopic treatment for vesico-ureteric reflux". Eur. Urol., 27: 76, 1995.

35. LACOMBE, A.: "Ureterovesical reimplantation after failure of endoscopic treatment of reflux by submucosal injection of polytef paste". Eur. Urol., 17: 318, 1990.

36. DODAT, H.; TAKVORIAN, P.: "Treatment of vesicoureteral reflux in children by endoscopic injection of Teflon. Review of 2 years of experience". Eur. Urol., 17: 304, 1990.

37. SAUVAGE, P.; GEISS, S.; SAUSSINE, C. y cols.: "Analysis and perspectives of endoscopic treatment of vesicoureteral reflux in children with a 20 -month follow-up". Eur. Urol., 17: 310, 1990.

38. SCHULMAN, C.C.; PAMART, D.; HALL, M. y cols.: "Vesicoureteral reflux in children: Endoscopic treatment". Eur. Urol., 17: 314, 1990.

39. DAVIES, N.; ATWELL, J.D.: "Primary vesicoureteric reflux: Treatment with subureteric injection of Polytef paste". Br. J. Urol., 67: 536, 1991.

40. BHATTI, H.A.; KHATTAK, H.; BOSTON, V.E.: "Efficacy and causes of failure of endoscopic subureteric injection of Teflon in the treatment of primary vesicoureteric reflux". Br. J. Urol., 71: 221, 1993.

41. OZYAVUZ, R.; OZGUR, G.K.; YUZUNCU, A.K.: "Subureteric polydimethylsiloxane injection in the treatment of vesicoureteric reflux". Int. Urol. Nephrol., 30: 123, 1998.

42. REUNANEN, M.: "Endoscopic collagen injection: Its limits in correcting vesico-ureteral reflux in duplicated ureters”. Eur. Urol., 31: 243, 1997.
43. YÜCEL, S.; TARCAN, T.; SIMSEK, F.: "Durability of a single successful endoscopic polytetrafluoroethylene injection for primary vesicoureteral reflux: 14 year follow up results". J. Urol., 178: 265, 2007.

44. DAWRANT, M.J.; NOCHIPARAMBIL, M.; PURI, P.: "Endoscopic treatment for high grade vesiureteral reflux in infants". J. Urol., 176: 1847, 2006.

45. PURI, P.; NOCHIPARAMBIL, M.; MENEZES, M. y cols.: "Endoscopic treatment of moderate and high grade vesicouretral reflux in infants using dextranomer/ hyaluronic acid". J. Urol., 178: 1714, 2007.

*46. PURI, P.; PIRKER, M.; NOCHIPARAMBIL, M. y cols.: "Subureteral dextranomer/hyaluronic acid injection as first line treatment in the management of high grade vesicoureteral reflux". J. Urol., 176: 1856, 2006.

47. MENEZES, M.; PURI, P.: "The role of endoscopic treatment in the management of grade $\mathrm{V}$ primary vesicoureteral reflux". Eur. Urol., 10: 1016, 2007.

48. YU, R.; ROTH, D.: "Treatment of vesicoureteral reflux using endoscopic injection on nonanimal stabiled hyaluronic acid/dextranomer gel: Initial experience in pediatrics patients by a single surgeon". Pediatrics, 118 : 698, 2006.

49. O'DONNELL, B.; PURI, P.: "Treatment of vesicoureteric reflux by endoscopic injection of Teflon". Br. Med. J. (Clin. Res. Ed.), 289: 7, 1984.

*50. LACKGREN, G.; WAHLIN, N.; SKOLDENBERG, E. y cols.: "Long-term follow up of children treated with dextranomer/ hyaluronic acid copolymer for vesicoureteral reflux". J. Urol., 166: 1887, 2001.

51. CAPOZZA, N.; CAIONE, P.: "Dextranomer/hyaluronic acid copolymer implantation for vesicoureteral reflux: A randomized comparision with antibiotic prophylaxis". J. Pediatr., 140: 230, 2002.

52. CAPOZZA, N.; LAIS, A.; MATARAZZO, E. y cols $\therefore$ "Influence of voiding dysfunction on the outcome of endoscopic treatment for vesicoureteral reflux". J. Urol., 168: 1695, 2002.

53. ROUTH, J.C.; VANDERSTEEN, D.R.; PFEFFERLE, H. y cols.: "Single center experience with endoscopic management of vesicoureteral reflux in children". J. Urol., 175: 1889, 2006.

54. PYLKKÄNEN, J.; VILSKA, J.; KOSKIMIES, O.: "The value of level diagnosis of childhood urinary tract infection in predicting renal injury". Acta Paediatr. Scand., 70: 879, 1981.

55. LINNE, T.; FITURI, O.; ESCOBAR-BILLING, R. y cols.: "Functional parameters and 99m-technetium dimercaptosuccinic acid scan in acute pyelonephritis". Pediatr. Nephrol., 8: 694, 1994.

56. NGUYEN, H.T.; BAUER, S.B.; PETERS, C.A. y cols.: "99m Technetium dimercapto-succinic acid renal scintigraphy abnormalities in infants with sterile high grade vesicoureteral reflux". J. Urol., 164: 1674, 2000.

57. ORSOLA DE LOS SANTOS, A.; CAFFARATTI SFULCINI, J.; GARAT BARREDO, J.M.: "Valor de la gammagrafía renal con DMSA en las pielonefritis agudas en niños". Urol. Integr. Invest., 2: 192, 1997.

58. PARKHOUSE, H.F.; GODLEY, M.L.; COOPER, J. y cols.: "Renal imaging with 99Tcm-labelled DMSA in the detection of acute pyelonephritis: An experimental study in the pig". Nucl. Med. Commun., 10: 63, 1989.

59. MAJD, M.; RUSHTON, H.G.: "Renal cortical scinti- 
graphy in the diagnosis of acute pyelonephritis". Semin. Nucl. Med., 22: 98, 1992.

60. WALLIN, L.; BAJC, M.: "The significance of vesicoureteric reflux on kidney development assessed by dimercaptosuccinate renal scintigraphy". Br. J. Urol., 73: 607, 1994.

61. MELIS, K.; VANDEVIVERE, J.; HOSKENS, C. y cols.: "Involvement of the renal parenchyma in acute urinary tract infection: The contribution of $99 \mathrm{mTc}$ dimercaptosuccinic acid scan”. Eur. J. Pediatr., 151: 536, 1992.

62. JAKOBSSON, B.; BERG, U.; SVENSSON, L.: "Renal scarring after acute pyelonephritis". Arch. Dis. Child, 70: 111, 1994

63. GOLDARICH, N.P.; GOLDARICH, I.H.: "Update on dimercaptosuccinic acid renal scaning in children with urinary tract infection". Pediatr. Nephrol., 9: 21, 1995.

64. RUSHTON, H.G.; MAJD, M.; JANTAUSCH, B. y cols .: "Renal scarring following reflux and nonreflux pyelonephritis in children: Evaluation with 99mtechnetium- dimercaptosuccinic acid scintigraphy". J. Urol., 147: 1327, 1992.

65. RUSHTON, H.G. Jr.: "Vesicoureteral reflux-new concepts and techniques". J. Urol., 157: 1414, 1997.

66. JAKOBSSON, B.; JACOBSON, S.H.; HJALMAS, K.: "Vesicoureteric reflux and other risk factors for renal damage: Identification of high and low risk children". Acta Paediatr. Suppl., 88: 31, 1999.

67. SLOVIS, T.L.: "Is there a single most appropriate imaging workup of a child with an acute febrile urinary tract infection?". Pediatr. Radiol., 1: 46, 1995.

68. HOBERMAN, A.; CHARRON, M.; HICKEY, R.W. y cols.: "Imaging studies after a first febrile urinary tract infection in young children". N. Engl. J. Med., 348: 195, 2003.

69. SWERKERSSON, S.; JODAL, U.; SIXT, R. y cols.: "Relationship among vesicoureteral reflux, urinary tract infection and renal damage in children". J. Urol., 178: 647, 2007.

70. ELO, J.; TALLGREN, L.G.; ALFTHAN, O. y cols.: "Character of urinary tract infections and pyelonephritic renal scarring after antireflux surgery". J. Urol., 129: 343, 193.

71. BIRMINGHAM REFLUX STUDY GROUP: "Prospective trial of operative versus nonoperative treatment of severe vesicoureteric reflux in children: Five years observation”. Br. Med. J. (Clin. Res. Ed.), 295: 237, 1987.

**72. OLBING, H.; CLAESSON, I.; EBEL, K.D. y cols.: "Renal scars and parenchymal thinning in children with vesicoureteral reflux: A 5-year report of the International Reflux Study in Children (European branch)". J. Urol., 148: 1653, 1992.

**73. WEISS, R.; DUCKETT, J.; SPITZER, A.: "Results of a randomized clinical trial of medical versus surgical management of infants and children with grades III and IV primary vesicoureteral reflux (United States). The International Reflux Study in Children”. J. Urol., 148: 1667, 1992.

74. WEBSTER, R.I.; SMITH, G.; FARNSWORTH, R.H. y cols.: "Low incidence of new renal scars after ureteral reimplantation for vesicoureteral reflux in children: A prospective study". J. Urol., 163: 1915, 2000.
75. PIEPSZ, A.; TAMMINEN-MOBIUS, T.; REINERS, C. y cols.: "Five-year study of medical or surgical treatment in children with severe vesico-ureteral reflux dimercaptosuccinic acid findings. International Reflux Study Group in Europe”. Eur. J. Pediatr., 157: 753, 1998.

76. AGGARWAL, V.K.; VERRIER JONES, K.; ASSCHER, A.W. y cols.: "Covert bacteriuria: Long term follow up". Arch. Dis. Child, 66: 1284, 1991.

77. BELLINGER, M.F.; DUCKETT, J.W.: "Vesicoureteral reflux: A comparison of non-surgical and surgical management". Contrib. Nephrol., 39: 81, 1984.

78. BEN-AMI, T.; SINAI, L.; HERTZ, M. y cols.: "Vesicoureteral reflux in boys: Review of 196 cases". Radiology, 173: 681, 1989.

79. BURGE, D.M.; GRIFFITHS, M.D.; MALONE, P.S. y cols.: "Fetal vesicoureteral reflux: Outcome following conservative postnatal management". J. Urol., 148: 1743, 1992.

80. CARDIFF-OXFORD BACTERIURIA STUDY GROUP: "Sequelae of covert bacteriuria in schoolgirls. A 4-year follow-up study". Lancet, 1: 889, 1978.

81. EDWARDS, D.; NORMAND, I.C.; PRESCOD, N. y cols.: "Disappearance of vesicoureteric reflux during long-term prophylaxis of urinary tract infection in children”. Br. Med. J., 2: 285, 1977.

82. HOMSY, Y.L.; NSOULI, I.; HAMBURGER, B. y cols.: "Effects of oxybutynin on vesicoureteral reflux in children". J. Urol., 134:1168, 1985.

83. HUSMANN, D.A.; ALLEN, T.D.: "Resolution of vesicoureteral reflux in completely duplicated systems: Fact or fiction?". J. Urol., 145: 1022, 1991.

84. JAKOBSEN, B.E.; GENSTER, H.; OLESEN, S. y cols.: "Vesico-ureteral reflux in children". Br. J. Urol., 49: 119, 1977.

85. KOFF, S.A.; MURTAGH, D.S.: "The uninhibited bladder in children: Effect of treatment on recurrence of urinary infection and on vesicoureteral reflux resolution". J. Urol., 130: 1138, 1983.

86. SCHOLTMEIJER, R.J.: "Problems in the diagnosis and treatment of children with urologic disorders". Ned. Tijdschr. Geneeskd., 132: 849, 1988.

87. SHAH, K.J.; ROBINS, D.G.; WHITE, R.H.: "Renal scarring and vesicoureteric reflux". Arch. Dis. Child., 53: 210,1978

**88. SMELLIE, J.M.; JODAL, U.; LAX, H. y cols.: "Outcome at 10 years of severe vesicoureteric reflux managed medically: Report of the International Reflux Study in Children". J. Pediatr., 139: 656, 2001.

*89. BEETZ, R.; SCHULTE-WISSERMANN, H.; TROGER, J. y cols.: "Long-term follow-up of children with surgically treated vesicorenal reflux: Postoperative incidence of urinary tract infections, renal scars and arterial hypertension. "Eur. Urol., 16: 366, 1989.

90. CHOI, H.; OH, S.J.; SO, Y. y cols.: "No further development of renal scarring after antireflux surgery in children with primary vesicoureteral reflux: Review of the results of 99mtechnetium dimercapto-succinic acid renal scan". J. Urol., 162: 1189, 1999.

91. LACKGREN, G.; WAHLIN, N.; STENBERG, A.: "Endoscopic treatment of children with vesico-ureteric reflux". Acta Paediatr. Suppl., 88: 62, 1999.

92. GARIN, E.; OLAVARIA, F.; GARCÍA, V. y cols.: "Cli- 
nical significance of primary vesicoureteral reflux and urinary prophylaxis after acute pyelonephritis: A multicenter, randomizad, controlled study". Pediatrics, 117: 626, 2006.

93. LYON, R.P.: "Renal arrest". J. Urol., 109: 707, 1973.

94. REDMAN, J.F.; SCRIBER, L.J.; BISSADA, N.K.: "Apparent failure of renal growth secondary to vesicoureteral reflux". Urology, 3: 704, 1974.

95. McRAE, C.U.; SHANNON, F.T.; UTLEY, W.L.: "Effect on renal growth of reimplantation of refluxing ureters". Lancet, 1: 1310, 1974.

96. PINTER, A.B.; JASZAI, V.; DOBER, I.: "Medical treatment of vesicoureteral reflux detected in infancy". J. Urol., 140: 121, 1988.

97. HERNDON, C.D.; McKENNA, P.H.; KOLON, T.F. y cols.: "A multicenter outcomes analysis of patients with neonatal reflux presenting with prenatal hydronephrosis". J. Urol., 162: 1203, 1999.

98. OLBING, H.; HIRCHE, H.; KOSKIMIES, O. y cols.: $\mathrm{H}$. "Renal growth in children with severe vesicoureteral reflux: 10-year prospective study of medical and surgical treatment: The International Reflux Study in Children (European branch)". Radiology, 216: 731, 2000.

99. BERG, U.B.: "Long-term followup of renal morphology and function in children with recurrent pyelonephritis". J. Urol., 148: 1715, 1992.

**100. SMELLIE, J.M.; BARRATT, T.M.; CHANTLER, C. y cols.: "Medical versus surgical treatment in children with severe bilateral vesicoureteric reflux and bilateral nephropathy: A randomised trial". Lancet, 357: 1329, 2001.

101. POULSEN, E.U.; JOHANNESEN, N.L.; NIELSEN, J.B. y cols.: "Vesico-ureteral reflux. II. The longterm outcome of kidney function in non-surgical treatment". Scand. J. Urol. Nephrol. Suppl., 125: 29, 1989.

102. POHL, H.G.; RUSHTON, H.G.; PARK, J.S. y cols.: "Adjunctive oral corticosteroids reduce renal scarring: The piglet model of reflux and acute experimental pyelonephritis". J. Urol., 162: 815, 1999.

103. BEETZ, R.; MANNHARDT, W.; FISCH, M. y cols.: "Long-term followup of 158 young adults surgically treated for vesicoureteral reflux in childhood: The ongoing risk of urinary tract infections". J. Urol., 168: 704, 2002.

104. HANSON, E.; HANSSON, S.; JODAL, U.: "Trimethoprimsulphadiazine prophylaxis in children with vesicoureteric reflux". Scand. J. Infect. Dis., 21: 201, 1989.

105. MANSFIELD, J.T.; SNOW, B.W.; CARTWRIGHT, P.C. y cols.: "Complications of pregnancy in women after childhood reimplantation for vesicoureteral reflux: An update with 25 years of follow up". J. Urol., 154: 787, 1995

106. LEWIS, M.A.: "Report of the Paediatric Renal Registry”. In: Answell D, Feest T. The Second Annual Report of the UK Renal registry. Bristol Renal Association, pag., 157-158, 1999.

107. WALLACE, D.M.; ROTHWELL, D.L.; WILLIAMS, D.I.: "The longterm follow-up of surgically treated vesicoureteric reflux". Br. J. Urol., 50: 479, 1978.

108. LENAGHAN, D.; WHITAKER, J.G.; JENSEN, F. y cols.: "The natural histiry of reflux and long-term effects of reflux on the kidney". J. Urol., 115: 728, 1976.
109. NIELSEN, J.B.; FROKIAER, J.; REHLING, M. y cols.: "A 14-year follow-up of conservative treatment for vesico-ureteric reflux". BJU Int., 86: 502, 2000.

110. KOHLER, J.R.; TENCER, J.; THYSELL, H. y cols.: "Long-term effects of reflux nephropathy on blood pressure and renal function in adults". Nephron. Clin. Pract., 93: 35, 2003.

111. GOONASEKERA, C.D.; DILLON, M.J.: "Hypertension in reflux nephropathy". BJU Int., 3: 1, 1999.

112. ZUCCHELLI, P.; GAGGI, R.: "Vesicoureteral reflux and reflux nephropathy in adults". Contrib. Nephrol., 61: 210, 1988

113. KINCAID-SMITH, P.; BECKER, G.: "Reflux nephropathy and chronic atrophic pyelonephritis: A review". J. Infect. Dis., 138: 774, 1978.

114. DWOSKIN, J.Y.; PERLMUTTER, A.D.: "Vesicoureteral reflux in children: A computerized review". J. Urol., 109: 888, 1973.

115. POLITO, C.; LA MANNA, A.; CAPACCHIONE, A. y cols.: "Height and weight in children with vesicoureteric reflux and renal scarring". Pediatr. Nephrol., 10: 564, 1996.

116. SMELLIE, J.M.; PREECE, M.A.; PATON, A.M.: "Normal somatic growth in children receiving low-dose prophylactic co-trimoxazole". Eur. J. Pediatr., 140: 301, 1983.

117. MERRELL, R.W.; MOWAD, J.J.: "Increased physical growth after successful antireflux operation". J. Urol., 122: 523,1979

118. POLITO, C.; MARTE, A.; ZAMPARELLI, M. y cols.: "Catch-up growth in children with vesicoureteric reflux". Pediatr. Nephrol., 11: 164, 1997.

119. ASTLEY, R.; CLRACK, R.C.; CORKERY, J.J. y cols.: "Prospective trial of operative versus nonoperative treatment of severe vesicoureteric reflux in children: Two years observation in 96 children". BMJ, 287: 171, 1983.

120. ASTLEY, R.; CLARK, R.C.; CORKERY, J.J. y cols.: "Prospective trial of operative versus nonoperative treatment of severe vesicoureteric reflux in children: Five years observation in 96 children". BMJ, 295: 237, 1987.

121. ORIKASA, S.: "A new antireflux operation". Eur. Urol., 17: 330, 1974.

122. BURBIGE, K.A.; MILLER, M.; CONNOR, J.P.: "Extravesical ureteral reimplantation: Results in 128 patients". J. Urol., 155: 1721, 1996.

123. DODAT, H.; VALMALLE, A.F.; WEIDMANN, J.D. y cols.: "Endoscopic treatment of vesicorenal reflux in children. Five-year assessment of the use of Macroplastique". Prog. Urol., 8: 1001, 1998.

124. KING, P.A.; GOLLOW, I.: "The endoscopic correction of vesico-ureteric reflux". Aust. NZ. J. Surg., 58: 569, 1988.

125. SWEENEY, L.E.; THOMAS, P.S.: "Evaluation of subureteric Teflon injection as an antireflux procedure". Ann. Radiol. (Paris), 30: 478, 1987.

126. DEWAN, P.A.: "Re: Evaluation of polydimethylsiloxane as an alternative in the endoscopic treatment of vesicoureteral reflux". J. Urol., 153: 1644, 1995.

127. KAMINETSKY, J.C.; HANNA, M.K.: "Endoscopic treatment of vesicoureteral reflux in children with neurogenic bladders". Urology, 37: 244, 1991. 
128. FREY, P.; LUTZ, N.; JENNY, P. y cols.: "Endoscopic subureteral collagen injection for the treatment of vesicoureteral reflux in infants and children". J. Urol., 154: 804, 1995.

129. GÓMEZ FRAILE, A.; ARANSAY BRANTOT, A.; LÓPEZ VÁZQUEZ, Y.F.: "Tratamiento endoscópico del reflujo vésico-ureteral en niños menores de un año". Urol. Integr. Invest., 7: 494, 2002.

130. EL-KHATIB, M.,; PACKHAM, D.K.; BECKER, G.J. y cols.: "Pregnancy-related complications in women with reflux nephropathy". Clin. Nephrol., 41: 50, 1994.

131. PURI, P.; NINAN, G.K.; SURANA, R.: "Subureteric Teflon injection (STING). Results of a European survey". Eur. Urol., 27: 71, 1995.

132. STENBERG, A.; LACKGREN, G.: "A new bioimplant for the endoscopic treatment of vesicoureteral reflux: Experimental and short-term clinical results". J. Urol., 154: 800, 1995

133. DIETZ, H.G.; SCHMIDT, A.; BADER, J.B. y cols.: "The Politano-Leadbetter antireflux plasty. Investigation of complications in 245 children”. Eur. J. Pediatr. Surg., 6: 277, 1996.

134. VUCKOV, S.; NIKOLIC, H.; KVESIC, A. y cols.: "Our experience in the treatment of the vesico-ureteral reflux with Lich-Gregoir antireflux surgical procedure". Eur. J. Pediatr. Surg., 9: 33, 1999.

135. LEISSNER, J.; ALLHOFF, E.P.; WOLFF, W. y cols.: "The pelvic plexus and antireflux surgery: Topographical findings and clinical consequences". J. Urol., 165: 1652, 2001.

136. LIPSKI, B.A.; MITCHELL, M.E.; BURNS, M.W.: "Voiding dysfunction after bilateral extravesical ureteral reimplantation”. J. Urol., 159: 1019, 1998.

137. MINEVICH, E.; ARONOFF, D.; WACKSMAN, J. y cols.: "Voiding dysfunction after bilateral extravesical detrusorrhaphy". J. Urol., 160: 1004, 1998.

138. ROSS, J.H.; KAY, R.; NASRALLAH, P.: "Contralateral reflux after unilateral ureteral reimplantation in patients with a history of resolved contralateral reflux". J. Urol., 154: 1171, 1995.

139. KUMAR, R.; PURI, P.: "Newly diagnosed contralateral reflux after successful unilateral endoscopic correction: Is it due to the pop-off mechanism?". J. Urol., 158: 1213, 1997.

140. MINEVICH, E.; WACKSMAN, J.; LEWIS, A.G. y cols.: "Incidence of contralateral vesicoureteral reflux following unilateral extravesical detrusorrhaphy (ureteroneocystostomy)". J. Urol., 159: 2126, 1998.

141. SARGIN, S.Y.; ERGEN, A.; OZEN, H. A. y cols.: "The fate of contralateral ureter after ipsilateral reimplantation in unilateral vesicoureteric reflux". Int. Urol. Nephrol., 23: 31, 1991.

142. CAIN, M.P.; HUSMANN, D.A.; McLAREN, R.H. y cols.: "Continuous epidural anesthesia after ureteroneocystostomy in children”. J. Urol., 154: 791, 1995.
143. PARK, J.M.; HOUCK, C.S.; SETHNA, N.F. y cols.: "Ketorolac suppresses postoperative bladder spasms after pediatric ureteral reimplantation". Anesth. Analg., 91: 11, 2000.

144. EL-GHONEIMI, A.; DEFFARGES, C.; HAN-KARD, R. y cols.: "Intravesical morphine analgesia is not effective after bladder surgery in children: Results of a randomied double-blind study". J. Urol., 168: 694, 2002.

145. SO, E.P.; BROCK, W.A.; KAPLAN, G.W.: "Ureteral reimplantation without catheters". J. Urol., 125: 551, 1981.

146. FORT, K.F.; SELMAN, S.H.; KROPP, K.A.: "A retrospective analysis of the use of ureteral stents in children undergoing ureteroneocystotomy”. J. Urol., 129: 545, 1983.

147. REMZI, D.; OZEN, H.A.; ERKAN, I. y cols.: "15 years of experience in the surgical treatment of vesicoureteral reflux in children". Turk. J. Pediatr., 26: 175, 1984.

148. BRANDELL, R.A.; BROCK, J.W. 3rd.: "Ureteral reimplantation: Postoperative management without catheters". Urology, 42: 705, 1993.

149. PURI, P.: "Endoscopic correction of vesicoureteral reflux”. Curr. Opin. Urol., 10: 593, 2000.

150. GRANATA, C.; BUFFA, P.; DI ROVASENDA, E. y cols.: "Treatment of vesico-ureteric reflux in children with neuropathic bladder: A comparison of surgical and endoscopic correction”. J. Pediatr. Surg., 34: 1836, 1999.

151. DIRECCIÓN GENERAL DE SALUD PÚBLICA.: "Guía de las indicaciones para la correcta solicitud de pruebas de diagnóstico por imagen”. Ed. C. Europeas, Madrid: Ministerio de Sanidad y Consumo, pp. 19-33, 2001.

152. ZERIN, J.M.; SHULKIN, B.: "Postprocedural symptoms in children who undergo imaging studies of the urinary tract: Is it the constrast material or the catheter?". Radiology, 182: 727, 1992.

153. ELDER, J.S.: "Guidelines for consideration for surgical repair of vesicoureteral reflux". Curr. Opin. Urol., 10: $579,2000$.

154. GOODING, C.; BERDON, W.E.; BRODEUR, A. y cols.: "Adverse reactions to intravenous pyelography in children". AJR, 123: 802, 1975.

155. BISSET, G.S. 3rd.; STRIFE, J.L.; DUNBAR, J.S.: "Urography and voiding cystourethrography: Findings in girls with urinary tract infection". AJR, 148: 479, 1987.

156. ABRAMS, P.H.; FREEMAN, R.B.; ANDERSTRÖM, C. y cols.: "Tolterodine a new antimuscarinic agent: As effective but better tolerated than oxybutynin in patients with overactive bladder". Br. J. Urol., 81: 801, 1998.

157. BOLDUC, S.; UPADHYAY, J.; PAYTON, J. y cols.: "The use of tolterodine in children after oxybutynin faliure”. BJU Int., 91: 398, 2003. 